\title{
Halo Sign and Eosinophilia: What Is Your Diagnosis?
}

\section{Florent Thevenet ${ }^{\mathrm{a}, \mathrm{b}}$ Guillaume Beltramo ${ }^{\mathrm{a}-\mathrm{c}}$ Marjolaine Georges ${ }^{\mathrm{a}, \mathrm{c}}$ Clément Foignot $^{\mathrm{a}, \mathrm{c}}$ Maximilien Spanjaard ${ }^{\mathrm{a}, \mathrm{c}}$ Philippe Bonniaud ${ }^{\mathrm{a}-\mathrm{c}}$}

a Service de Pneumologie et Soins Intensifs Respiratoires, Centre Hospitalo-Universitaire de Bourgogne, Dijon, France; ' INSERM, LNC UMR866, LipSTIC LabEx team, Dijon, France;

'Université Bourgogne Franche-Comté, Dijon, France

\section{Keywords}

Halo sign · Eosinophilia · Larva migrans

\section{Abstract}

Blood eosinophilia associated with transient and migrating nodules with a halo sign on chest computed tomography scan should suggest larva migrans related to toxocariasis or ascaris suum.

Fig. 1. a, b Multiple nodular lesions with halo sign and ground-glass opacity nodules. c, d Magnification of ground-glass opacities with halo sign.
A 44-year-old never-smoker was evaluated for recent isolated dry cough and urticaria. Blood cell analysis revealed an eosinophil count of $2,080 / \mu$ l. Antinuclear antibodies and antineutrophil cytoplasmic antibodies were negative. Chest radiograph was normal. High-resolution computed tomography (CT) showed 8 ground-glass opacities with ill-defined margins and 4 nodules with halos measuring from 12 to $25 \mathrm{~mm}$ with a distribution predominantly in the lower and peripheral part of both lungs
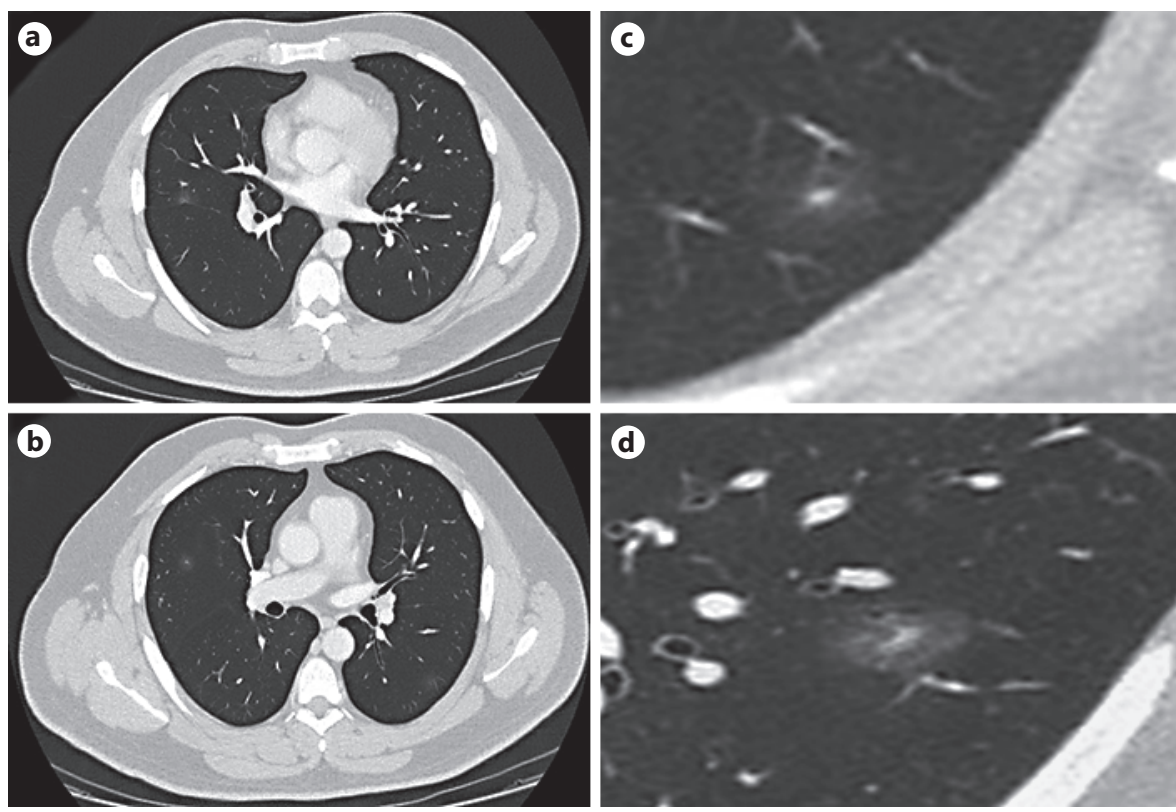

(C) 2017 S. Karger AG, Basel

E-Mail karger@karger.com

www.karger.com/res
Philippe Bonniaud, $\mathrm{MD}, \mathrm{PhD}$

Service de Pneumologie et Soins Intensifs Respiratoires

Centre Hospitalo-Universitaire de Bourgogne

14 rue Gaffarel, FR-21000 Dijon (France)

E-Mail philippe.bonniaud@chu-dijon.fr 
(Fig. 1). A diagnosis of larva migrans was suspected. Toxocara antibodies were strongly positive. We started treatment with ivermectin. Our patient's clinical symptoms improved within 2 weeks. One month later, followup CT showed a migration of the nodules. The eosinophil count normalized within 4 months.

Humans are an accidental host of Toxocara, which normally infects dogs (Toxocara canis) and cats (Toxocara cati). In western countries, $2-5 \%$ of all adults in urban areas and $14-37 \%$ of all adults in rural areas are seropositive for Toxocara. "Larva migrans" is associated with acute eosinophilic inflammation and allergic manifestations. With regard to the lung, patients may suffer from cough, dyspnea, asthma, or acute bronchiolitis, and infrequently infiltrative pneumonitis or acute respiratory distress.

Beyond the serological diagnosis, the radiological findings are crucial to strengthen the diagnosis. Multiple nodules, consolidations, pleural effusions, and migrating ground-glass opacities have been described on chest CT. The peculiar transient nodules with a halo sign may migrate and have been specifically described in toxocariasis [1] and ascaris suum [2]. They are usually multiple, of variable size, and disseminated in the peripheral areas of the lungs [3]. Their presence seems to correlate with high eosinophilic blood levels [4].
References
1 Kang YR, Kim SA, Jeon K, et al: Toxocariasis as a cause of new pulmonary infiltrates. Int J Tuberc Lung Dis 2013;17:412-417.

2 Sakai S, Shida Y, Takahashi N, et al: Pulmonary lesions associated with visceral larva migrans due to Ascaris suum or Toxocara canis: imaging of six cases. AJR Am J Roentgenol 2006;186:1697-1702.
3 Mazur-Melewska K, Jonczyk-Potoczna K, Kemnitz P, Mania A, Figlerowicz M, Sluzewski W: Pulmonary presentation of Toxocara sp. infection in children. Pneumonol Alergol Pol 2015;83:250-255.

4 Hur JH, Lee IJ, Kim JH, et al: Chest CT findings of toxocariasis: correlation with laboratory results. Clin Radiol 2014;69:e285-e290.
Thevenet/Beltramo/Georges/Foignot/ Spanjaard/Bonniaud 